Lili Wang*

\title{
Crystal structure of $(E)-N^{\prime}-((1,6-$ dihydropyren-1-yl) methylene)isonicotinohydrazide - methanol (1/1), $\mathrm{C}_{24} \mathrm{H}_{19} \mathrm{~N}_{3} \mathrm{O}_{2}$
}

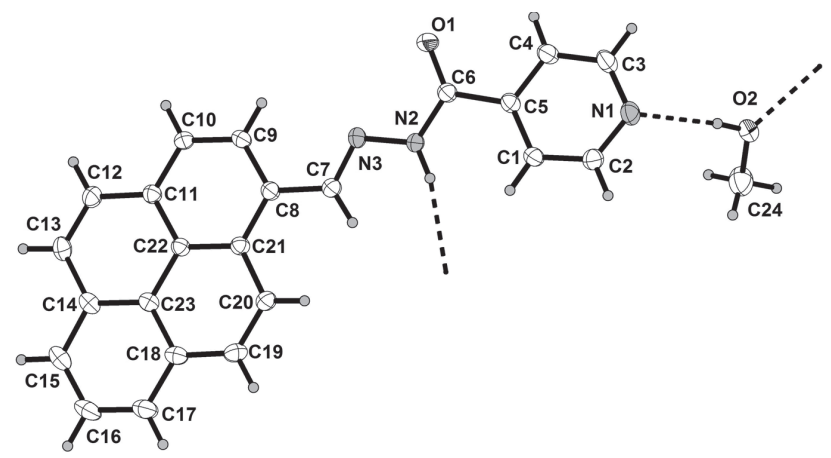

https://doi.org/10.1515/ncrs-2019-0370

Received May 26, 2019; accepted July 16, 2019; available online August 2, 2019

\section{Abstract \\ $\mathrm{C}_{24} \mathrm{H}_{19} \mathrm{~N}_{3} \mathrm{O}_{2}$, monoclinic, $P 22_{1} / c$ (no. 14), $a=11.0629$ (2) $\AA$, $b=10.4583(2) \AA, \quad c=16.0432(3) \AA, \quad \beta=92.373(2)^{\circ}$, $V=1854.59(7) \AA^{3}, Z=4, R_{\mathrm{gt}}(F)=0.0435, w R_{\mathrm{ref}}\left(F^{2}\right)=0.1269$, $T=116.2(3) \mathrm{K}$.}

\section{CCDC no.: 1940940}

The asymmetric unit of the cocrystal title structure is shown in the figure. Table 1 contains crystallographic data and Table 2 contains the list of the atoms including atomic coordinates and displacement parameters.

\section{Source of material}

All solvents and starting materials are commercially available and used without further treatment. The target compound was facilely synthesized by one-step condensation reaction according to reference [5].

Pyrene-1-carbaldehyde (1 mmol, $230 \mathrm{mg}$ ) was dispersed with appropriate amount of ethanol. Isonicotinohydrazide ( $1 \mathrm{mmol}, 137 \mathrm{mg}$ ) was dissolved with appropriate amount of absolute ethanol to obtain a colorless solution. Then the two solutions were added under stirring. The stirred mixture was refluxed for $4 \mathrm{~h}$ to appear yellow precipitation immediately.

*Corresponding author: Lili Wang, Henan University of Chinese Medicine, Zhengzhou 450046, P.R. China, e-mail: wllywlly2004@163.com
Table 1: Data collection and handling.

\begin{tabular}{|c|c|}
\hline Crystal: & Dark yellow cube \\
\hline Size: & $0.40 \times 0.20 \times 0.10 \mathrm{~mm}$ \\
\hline Wavelength: & Cu $K \alpha$ radiation (1.54184 ̊̊) \\
\hline$\mu:$ & $0.71 \mathrm{~mm}^{-1}$ \\
\hline Diffractometer, scan mode: & SuperNova, $\omega$ \\
\hline$\theta_{\max }$, completeness: & $71.6^{\circ},>99 \%$ \\
\hline$N(h k l)_{\text {measured }}, N(h k l)_{\text {unique }}, R_{\text {int }}:$ & $6518,3501,0.024$ \\
\hline Criterion for $I_{\text {obs }}, N\left(h k l l_{\mathrm{gt}}\right.$ : & $I_{\text {obs }}>2 \sigma\left(I_{\text {obs }}\right), 3098$ \\
\hline$N(\text { param })_{\text {refined }}:$ & 264 \\
\hline Programs: & $\begin{array}{l}\text { CrysAlis }^{\text {PRO }}[1] \text {, Olex2 [2], SHELX } \\
{[3,4]}\end{array}$ \\
\hline
\end{tabular}

Table 2: Fractional atomic coordinates and isotropic or equivalent isotropic displacement parameters $\left(\AA^{2}\right)$.

\begin{tabular}{lrrrr}
\hline Atom & $\boldsymbol{X}$ & $\boldsymbol{y}$ & $\boldsymbol{Z}$ & $\boldsymbol{U}_{\text {iso }} \boldsymbol{U}_{\text {eq }}$ \\
\hline O1 & $0.42268(9)$ & $0.10172(9)$ & $0.69591(7)$ & $0.0324(3)$ \\
N1 & $0.01551(11)$ & $0.11005(12)$ & $0.81951(7)$ & $0.0297(3)$ \\
N2 & $0.37482(10)$ & $0.31257(11)$ & $0.68270(7)$ & $0.0238(3)$ \\
H2 & 0.3228 & 0.3716 & 0.6915 & $0.029^{*}$ \\
N3 & $0.48052(10)$ & $0.34115(11)$ & $0.64438(7)$ & $0.0245(3)$ \\
C1 & $0.16219(12)$ & $0.26467(13)$ & $0.77868(8)$ & $0.0259(3)$ \\
H1 & 0.1851 & 0.3501 & 0.7760 & $0.031^{*}$ \\
C2 & $0.05517(13)$ & $0.23032(14)$ & $0.81389(9)$ & $0.0286(3)$ \\
H2A & 0.0076 & 0.2950 & 0.8351 & $0.034^{*}$ \\
C3 & $0.08805(13)$ & $0.01907(14)$ & $0.79100(9)$ & $0.0307(3)$ \\
H3 & 0.0634 & -0.0656 & 0.7954 & $0.037^{*}$ \\
C4 & $0.19749(13)$ & $0.04323(13)$ & $0.75535(8)$ & $0.0275(3)$ \\
H4 & 0.2450 & -0.0237 & 0.7371 & $0.033^{*}$ \\
C5 & $0.23513(12)$ & $0.16935(13)$ & $0.74738(8)$ & $0.0230(3)$ \\
C6 & $0.35336(12)$ & $0.19035(13)$ & $0.70646(8)$ & $0.0232(3)$ \\
C7 & $0.49086(12)$ & $0.45959(13)$ & $0.62524(8)$ & $0.0235(3)$ \\
H7 & 0.4305 & 0.5168 & 0.6391 & $0.028^{*}$ \\
C8 & $0.59554(12)$ & $0.50589(12)$ & $0.58216(8)$ & $0.0217(3)$ \\
C9 & $0.67768(12)$ & $0.41868(12)$ & $0.54982(8)$ & $0.0241(3)$ \\
H9 & 0.6650 & 0.3315 & 0.5570 & $0.029^{*}$ \\
C10 & $0.77645(12)$ & $0.45904(12)$ & $0.50781(8)$ & $0.0244(3)$ \\
H10 & 0.8295 & 0.3989 & 0.4871 & $0.029^{*}$ \\
C11 & $0.79845(12)$ & $0.58999(12)$ & $0.49563(8)$ & $0.0219(3)$ \\
C12 & $0.90103(12)$ & $0.63484(14)$ & $0.45253(8)$ & $0.0259(3)$ \\
H12 & 0.9555 & 0.5758 & 0.4324 & $0.031^{*}$ \\
C13 & $0.92043(13)$ & $0.76079(14)$ & $0.44049(8)$ & $0.0281(3)$ \\
H13 & 0.9879 & 0.7870 & 0.4122 & $0.034^{*}$
\end{tabular}


Table 2 (continued)

\begin{tabular}{lrrrr}
\hline Atom & $\boldsymbol{x}$ & $\boldsymbol{y}$ & $\boldsymbol{z}$ & $\boldsymbol{U}_{\text {iso }} \boldsymbol{U}_{\text {eq }}$ \\
\hline C14 & $0.83888(13)$ & $0.85459(13)$ & $0.47052(8)$ & $0.0262(3)$ \\
C15 & $0.85508(14)$ & $0.98650(14)$ & $0.45752(9)$ & $0.0325(3)$ \\
H15 & 0.9208 & 1.0148 & 0.4283 & $0.039^{*}$ \\
C16 & $0.77467(16)$ & $1.07425(14)$ & $0.48755(10)$ & $0.0374(4)$ \\
H16 & 0.7866 & 1.1609 & 0.4778 & $0.045^{*}$ \\
C17 & $0.67646(14)$ & $1.03539(14)$ & $0.53204(10)$ & $0.0335(3)$ \\
H17 & 0.6237 & 1.0960 & 0.5524 & $0.040^{*}$ \\
C18 & $0.65625(13)$ & $0.90504(13)$ & $0.54649(8)$ & $0.0263(3)$ \\
C19 & $0.55628(13)$ & $0.86126(13)$ & $0.59229(9)$ & $0.0281(3)$ \\
H19 & 0.5044 & 0.9208 & 0.6148 & $0.034^{*}$ \\
C20 & $0.53539(12)$ & $0.73495(13)$ & $0.60370(8)$ & $0.0251(3)$ \\
H20 & 0.4689 & 0.7097 & 0.6333 & $0.030^{*}$ \\
C21 & $0.61399(12)$ & $0.63889(12)$ & $0.57105(8)$ & $0.0208(3)$ \\
C22 & $0.71619(11)$ & $0.68008(12)$ & $0.52723(8)$ & $0.0205(3)$ \\
C23 & $0.73715(12)$ & $0.81372(12)$ & $0.51454(8)$ & $0.0223(3)$ \\
O2 & $-0.23393(9)$ & $0.05521(10)$ & $0.79425(7)$ & $0.0357(3)$ \\
H2B & -0.1638 & 0.0658 & 0.8113 & $0.054^{*}$ \\
C24 & $-0.26909(16)$ & $0.15720(19)$ & $0.74123(12)$ & $0.0464(4)$ \\
H24A & -0.2144 & 0.1631 & 0.6965 & $0.070^{*}$ \\
H24B & -0.2672 & 0.2356 & 0.7723 & $0.070^{*}$ \\
H24C & -0.3497 & 0.1424 & 0.7188 & $0.070^{*}$ \\
\hline & & & &
\end{tabular}

The product was filtered, washed several times with ethanol/ water (1:1) to obtain yellow powder. Yield: ca. $80 \%$.

The yellow powder $(0.05 \mathrm{mmol}, 17.5 \mathrm{mg})$ was dissolved in methanol/dichloromethane (4:1) $(10 \mathrm{~mL})$ under stirring to give a pale yellow solution. This solution was filtered and evaporated slowly at room temperature. After one week, yellow cube crystals appeared. Yield: ca. $60 \%$.

\section{Experimental details}

The $\mathrm{H}$ atoms were added geometrically using riding models and refined isotropically. Their $U_{\text {iso }}$ values were set to $1.2 U_{\text {eq }}$ of the parent $\mathrm{C}$ and $\mathrm{N}$ atoms, and $1.5 U_{\text {eq }}$ of the parent $\mathrm{O}$ atoms.

\section{Comment}

The application of fluorescent probe technology has been widely focused on by more and more scientists in biochemistry, analytical chemistry and medicine engineering in recent years [6-9]. The fluorescent probes based on pyrene derivatives have been reported $[5,10]$. We herein report a new pyrene-derived compound, which has good coordination ability to transition metal and rare-earth metal ions.

The asymmetric unit of the title compound contains a neutral molecule and a methanol solvent molecule. The bond length of C7-N3 is 1.2824(18) $\AA$, which indicate that the target compound was synthesized successfully. Methanol molecules link the adjacent molecules into a chain structure. Hydrogen bond length between $\mathrm{N} 1$ of pyridine ring and $\mathrm{O} 2$ on methanol hydroxyl group is 2.831 A. Hydrogen bond length between N2 of imine and 02 on methanol hydroxyl group is $3.009 \AA$ (cf. the figure).

\section{References}

1. Rigaku Oxford Diffraction: CrysAlis Pro Software system, version 1. 171. 38. 43f, Rigaku Oxford Diffraction (2015).

2. Dolomanov, O. V.; Bourhis, L. J.; Gildea, R. J.; Howard, J. A. K.; Puschmann, H.: OLEX2: a complete structure solution, refinement and analysis program. J. Appl. Crystallogr. 42 (2009) 339-341.

3. Sheldrick, G. M.: Crystal structure refinement with SHELXL. Acta Crystallogr. C71 (2015) 3-8.

4. Sheldrick, G. M.: SHELXT-integrated space-group and crystal-structure determination. Acta Crystallogr. A71 (2015) 3-8.

5. Zhou, Y.; Wang, F.; Kim, Y.; Kim, S.; Yoon, J.: $\mathrm{Cu}^{2+}$-selective ratiometric and "off-on" sensor based on the rhodamine derivative bearing pyrene group. Org. Lett. 11 (2009) 4442-4445.

6. Mondal, S.; Manna, S. K.; Maiti, K.; Maji, R.; Ali, S. S.; Manna, S.; Mandal, S.; Uddin, M. R.; Mahapatra, A. K.: Phenanthroline-fluorescein molecular hybrid as a ratiometric and selective fluorescent chemosensor for $\mathrm{Cu}^{2+}$ via FRET strategy: synthesis, computational studies and in vitro applications. Supramol. Chem. 29 (2017) 616-626.

7. Zhao, Y.; Zhang, X.-B.; Han, Z.-X.; Qiao, L.; Li, C.-Y.; Jian, L.-X.; Shen, G.-L.; Yu, R.-Q.: Highly sensitive and selective colormetric and off-on fluorescent chemosensor for $\mathrm{Cu}^{2+}$ in aqueous solution and living cells. Anal. Chem. 81 (2009) 7022-7030.

8. Yang, Y.; Zhao, Q.; Feng, W.; Li, F.: Luminescent chemodosimeters for bioimaging. Chem. Rev. 113 (2013) 192-270.

9. Huang, H.; Zhang, P.; Chen, H.; Ji, L.; Chao, H.: Comparison between polypyridyl and cyclometalated ruthenium(II) complexes: anticancer activities against 2D and 3D cancer models. Chem. Eur. J. 21 (2015) 715-725.

10. Manandhar, E.; Broome, J. H.; Myrick, J.; Lagrone, W.; Cragg, P. J.; Wallace, K. J.: A pyrene-based fluorescent sensor for $\mathrm{Zn}^{2+}$ ions: a molecular 'butterfly'. Chem. Commun. 47 (2011) 8796-8798. 